\title{
All printed transparent electrodes through an electrical switching mechanism: A convincing alternative to indium-tin-oxide, silver and vacuum
}

\author{
Larsen-Olsen, Thue Trofod; Søndergaard, Roar; Norrman, Kion; Jørgensen, Mikkel; Krebs, Frederik C
}

Published in:

Energy \& Environmental Science

Link to article, DOI:

$10.1039 / \mathrm{c} 2 \mathrm{ee} 23244 \mathrm{~h}$

Publication date:

2012

Document Version

Publisher's PDF, also known as Version of record

Link back to DTU Orbit

Citation (APA):

Larsen-Olsen, T. T., Søndergaard, R., Norrman, K., Jørgensen, M., \& Krebs, F. C. (2012). All printed transparent electrodes through an electrical switching mechanism: A convincing alternative to indium-tin-oxide, silver and vacuum. Energy \& Environmental Science, 5(11), 9467-9471. https://doi.org/10.1039/c2ee23244h

\section{General rights}

Copyright and moral rights for the publications made accessible in the public portal are retained by the authors and/or other copyright owners and it is a condition of accessing publications that users recognise and abide by the legal requirements associated with these rights.

- Users may download and print one copy of any publication from the public portal for the purpose of private study or research.

- You may not further distribute the material or use it for any profit-making activity or commercial gain

- You may freely distribute the URL identifying the publication in the public portal 


\title{
Environmental Science
}

\section{All printed transparent electrodes through an electrical switching mechanism: A convincing alternative to indium-tin-oxide, silver and vacuum}

\author{
Thue T. Larsen-Olsen, Roar R. Søndergaard, Kion Norrman, Mikkel Jørgensen and Frederik C. Krebs*
}

Received 22nd August 2012, Accepted 5th September 2012

DOI: $10.1039 / \mathrm{c} 2 \mathrm{ee} 23244 \mathrm{~h}$

\begin{abstract}
Here we show polymer solar cells manufactured using only printing and coating of abundant materials directly on flexible plastic substrates or barrier foil using only roll-to-roll methods. Central to the development is a particular roll-to-roll compatible post-processing step that converts the pristine and non-functional multilayercoated stack into a functional solar cell through formation of a charge selective interface, in situ, following a short electrical pulse with a high current density. After the fast post-processing step the device stack becomes active and all devices are functional with a technical yield and consistency that is compelling.
\end{abstract}

Although polymer and organic solar cells have been generally recognized for more than a decade and presented in a vision of low cost flexible solar panels with a thin outline and low cost, state-of-the art polymer solar cells are still handled in a glove box and employ rigid glass substrates, expensive indium-tin-oxide (ITO) and expensive or reactive metal electrodes evaporated under high vacuum. This reflects a developmental focus which has until now been mostly on increasing the solar cell efficiency ${ }^{1}$ while neglecting the decisive issues of stability ${ }^{2}$ and true scalability through the exclusive use of roll-to-roll processing and abundant materials with a low thermal budget. ${ }^{3,4}$ The transition from single laboratory devices to mass produced modules has been

Department of Energy Conversion and Storage, Technical University of Denmark, Frederiksborgvej 399, DK-4000 Roskilde, Denmark. E-mail: frkr@dtu.dk slow and it has proven difficult to transfer the high performance reported for laboratory devices to a larger scale. Often results for laboratory devices are represented by a single (or a few) successful experiment(s) with the values for the "hero" device being quoted. Mass produced polymer solar cells, however, are represented by the average which in reality is what can become useful. In such a case the technical yield and consistency become critically important and the robustness of materials and processes is paramount. The few documented cases where many polymer solar cells have been reported are based on ProcessOne ${ }^{5}$ which does fulfill the criteria of robustness and repeatability to an extent that many modules can be prepared with an even performance and integrated into demonstrators. In a recent example more than 10000 small credit card sized OPV modules based on ProcessOne were manufactured and integrated into a small flashlamp. ${ }^{6}$ ProcessOne comprise a PET-ITO-ZnO-P3HT:PCBMPEDOT:PSS-silver stack and present performances in the range of $1.5-2 \%$ in an identical module layout. ${ }^{6}$ In terms of materials usage however ProcessOne will never be competitive since it involves ITO that is arrived at by vacuum deposition followed by a time consuming lithographic process. ${ }^{7}$ The overall processing speed of the patterned ITO electrode is unlikely to exceed $1 \mathrm{~m} \mathrm{~min}^{-1}$ even with large industrial machinery. Apart from the processing of ITO, the material involves the very rare metal indium and possesses some less critical attributes such as brittleness and poor thermomechanical properties.

There has been a significant research effort aimed at replacing ITO, and while there have been some successful laboratory reports ${ }^{8-12}$ only few are directly compatible with large scale processing of

\section{Broader context}

The vision of polymer solar cells is a scalable, efficient and stable technology that can be prepared in large areas with a thin outline using ultrafast printing and coating methods that require little energy in the process and only abundant materials. State-of-the-art polymer and organic solar cells are far removed from this ideal situation and typically employ tiny areas processed through slow and time consuming vacuum steps on rigid substrates employing toxic processing conditions and elements with low abundance such as indium and silver. We present a simple solar cell stack that comprises only four printed and coated layers representing significant progress at all levels. We have thus moved from single cells to modules, from rigidity to flexibility, from spin coating to full roll-toroll processing in all steps, from glove box to ambient processing, thousands of units and have eliminated the use of indium, silver and vacuum while achieving comparable performance. We introduce a method whereby functionality is arrived at through in situ formation of a rectifying interface inside the finished stack. We demonstrate how this switching mechanism is fully scalable and we use it in a fully automated roll-to-roll process. We also elucidate the chemistry behind this generic mechanism as a new processing step. 
interconnected modules. The first successful example ${ }^{8}$ involved a nontransparent first electrode based on coated silver nanoparticles. Even if the performance was quite poor compared to ProcessOne it did show that an ITO-free future could be anticipated pending the right developments.

To rationalize our efforts towards replacing ITO, one must logically also consider the relatively low abundance and high thermal budget of silver which make its use just as critical as the use of indium. ${ }^{7}$ Silver, however, has the advantage over ITO of an exceptionally high conductivity enabling the use of much less material, if applied correctly as a very thin layer or a grid. In the ultimate case, however, even silver cannot be anticipated as being on the materials list unless it can be recycled efficiently.

When focusing on the functional part of the solar cell stack it comprises the active layer sandwiched between an electron and a hole selective layer where one or both of the charge selective layers may serve as electrode.

For the purpose of this study we introduce the generalized stack shown as the center piece in Fig. 1, comprising first-electrode/ZnO/ active layer/PEDOT:PSS/second-electrode, forming the functional basis for the solar cell module, as succeeding stacks can be serially connected by overlapping of their first- and second-electrode. In the ideal case the two electrodes are based on abundant materials. Here we utilize a compact module comprised of 16 serially connected solar cells which enables us to alleviate the use of both ITO and Ag, thus in essence presenting a metal electrode-free solar cell module. The chosen device structures were thus simply PEDOT:PSS/ZnO/active layer/PEDOT:PSS/graphite, while a series of modules using $\mathrm{Ag}$ instead of graphite as the second-electrode were also fabricated for comparison.

Returning to the scalability and processability of this device stack it is highly compatible with high speed printing at all levels, especially the front electrode including the $\mathrm{ZnO}$ layer could be processed at very high speed even under simple pilot line conditions as shown in Fig. 1. We were thus able to process the entire front electrode structure with the front PEDOT:PSS electrode being formulated for rotary screen printing enabling printing speeds with high accuracy at $>10 \mathrm{~m} \mathrm{~min}^{-1}$ (speed limited by the drier length of 2 $\mathrm{m})$. The slot-die coating of the $\mathrm{ZnO}$ nanoparticle ink could easily be coated at $10 \mathrm{~m} \mathrm{~min}^{-1}$ also with high accuracy (48 cell lines coated simultaneously, $3 \mathrm{~mm}$ wide and spaced by $1 \mathrm{~mm}$ ). The sheet resistivity of the pure PEDOT:PSS was $60 \mathrm{ohm}$ per square and sufficient for transport over the $2 \mathrm{~mm}$ wide active area with part of the $4 \mathrm{~mm}$ repeat covered by the thick printed interconnection (Fig. 2e). The electrode structure thus presents a significant alternative to ITO at all levels by being comparable in performance, while being abundant and processable in air without vacuum. The active layer was like the $\mathrm{ZnO}$ layer slot-die coated and the back PEDOT:PSS electrode was rotary screen printed in registry with the underlying layer structure (for modules the interconnects or grids were also rotary screen printed in registry). It is of significant importance to underline that the realization of the patterned multilayer stack was readily achievable using standard coating and printing techniques (pending an appreciable effort in ink formulation, printing/coating methodology and machine design) and as such it did not present inventive steps (only skill).

The testing of the multilayer stack as a solar cell however presented a non-functional device with a very low parallel resistance as shown in Fig. 2b (essentially a short circuit). At first this was ascribed to coating or printing irregularities that bridge the two electrodes through the thin active part of the solar cell thus creating a short circuit. However, the process is in essence identical to ProcessOne where large numbers of similar solar cell modules can be made reliably, and careful analysis revealed that processing faults were not the cause.

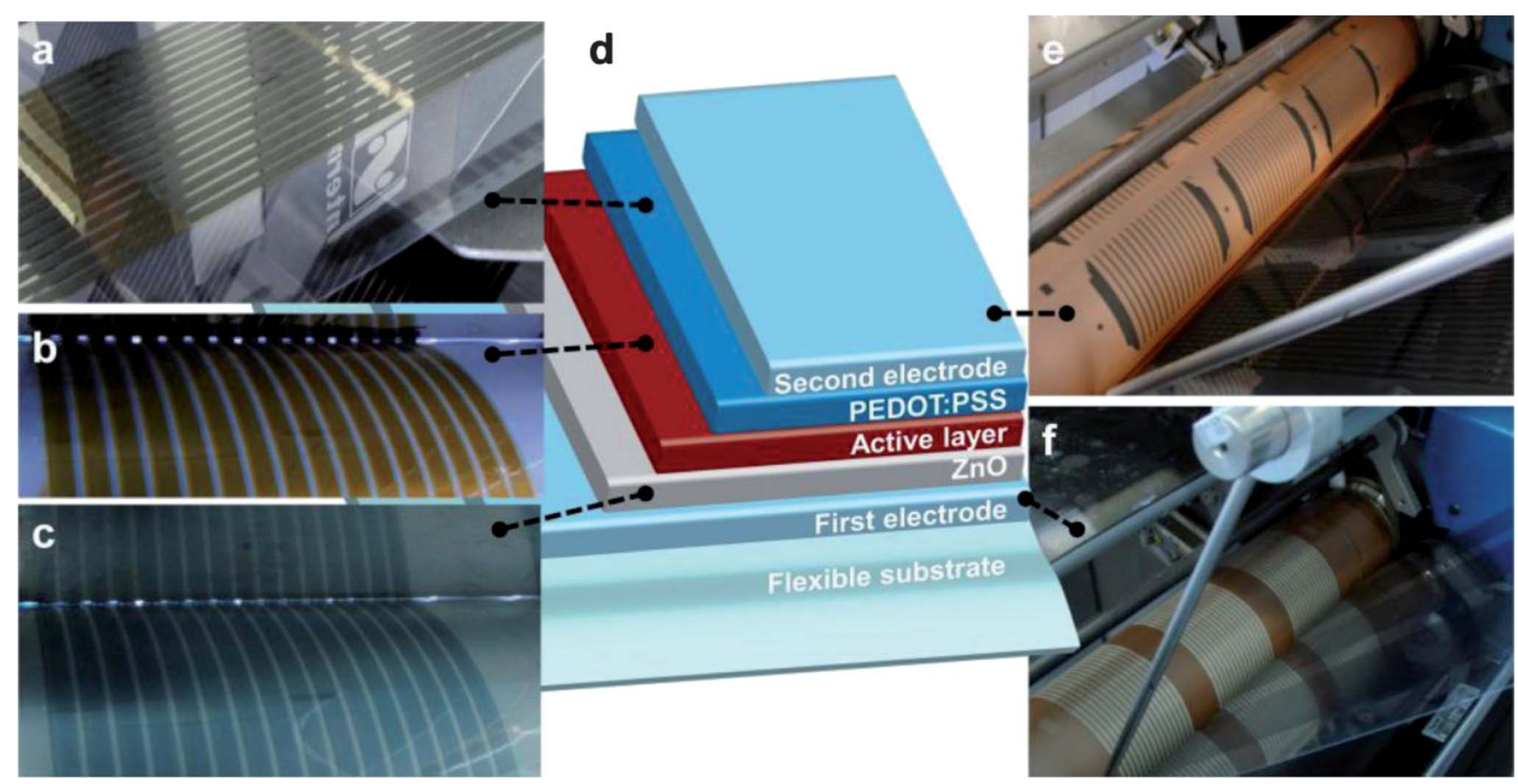

Fig. 1 The device structure (d) centrally surrounded by photographs of the R2R coating and printing, with (a) and (f) showing the rotary screen printing of the top and bottom PEDOT:PSS layers, respectively. (c) and (b) showing the slot-die coating of ZnO and P3HT:PCBM, respectively, while (e) shows the rotary screen printing of the graphite second-electrode. 


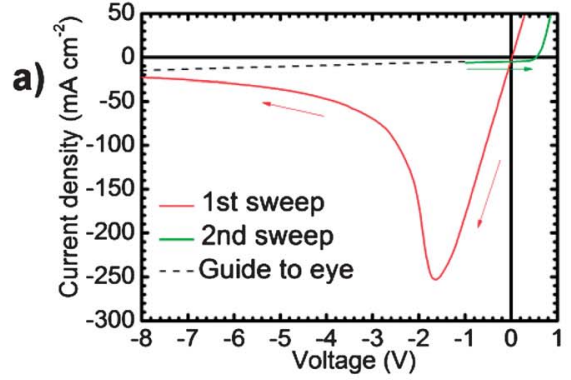

b)

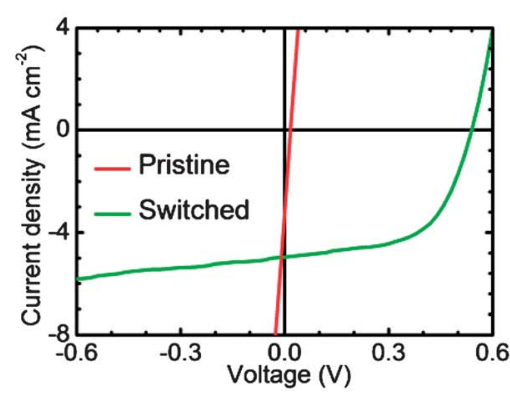

c)

Pristine device

e)

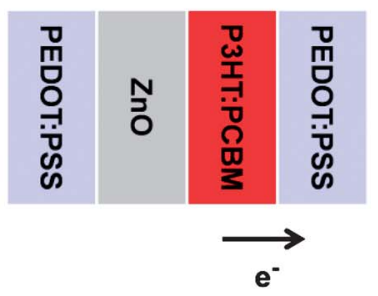

d)

\section{Switched device}

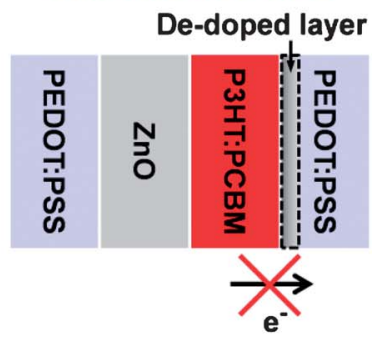

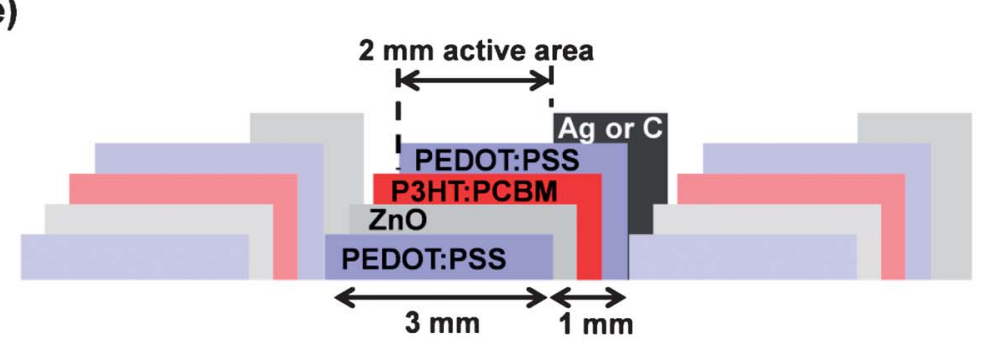

Fig. 2 Electrical characterization. (a) The $I-V$ characterization under simulated solar light, showing the initial bias sweep (red) going from $1 \mathrm{~V}$ to $-8 \mathrm{~V}$, followed by a forward sweep from $-1 \mathrm{~V}$ to $1 \mathrm{~V}$ (green). (b) A zoom-in on the active quadrant, showing the change in device performance. (c and d) show the layer stack before and after the de-doping has occurred. (e) shows a cross section of the device highlighting the active region and in the printed interconnection.

$\mathrm{ZnO}$ as a semi conductor is known to present interaction with atmospheric oxygen ${ }^{13}$ and can also be subject to proton doping. ${ }^{14}$ Both effects alter the transport properties of $\mathrm{ZnO}$ and can also be expected to be present in the stack we explore here. We found by studying very small area devices $\left(\leq 25 \mathrm{~mm}^{2}\right)$ that a high current density at a significant applied field presented an irreversible response vis-à-vis a report on the use of PEDOT:PSS as a write-once-readmany-times (WORM) memory material ${ }^{15-20}$ and $\mathrm{ZnO}$ as a reversible memory element. . $^{1,21,22}$

Fortunately, the device works exceptionally well after the short high current density-high electric field treatment as shown in Fig. 2b and it was thus a matter of characterizing the effect in order to make proper use of it in solar cell devices. The observation that a high negative bias dramatically alters the electrical performance is ascribed to the known permanent conductivity change in PEDOT:PSS films.

A switching mechanism was first demonstrated by Möller et al. ${ }^{15,16}$ and later adopted by others, ${ }^{17-20}$ in all cases for WORM devices. The exact nature of this bias induced phenomenon is still debated, but apart from de Brito et al. ${ }^{18}$ who ascribed the conductivity loss to a delamination caused by gases produced by hydrolysis, it is believed to be caused by de-doping of the PEDOT $\left(\right.$ PEDOT $^{+} \rightarrow$ PEDOT $^{0}$ ) molecules induced by charge injection, while the dedoped state is stabilized by the neutralization of the PSS counter-ions ( $\mathrm{PSS}^{-} \rightarrow$ PSS-H). ${ }^{16,19,23}$ Here we report for the first time that the de-doped PEDOT:PSS layer functions as a very efficient hole selective layer for solar cells. The proposed mechanism is, as illustrated in Fig. 2c and d, blocking of electron transport by the formation of a sufficiently thick de-doped PEDOT:PSS layer. The layer thus constitutes a thin region where the polythiophene is reduced and it thus behaves like an intrinsic semiconductor. The mechanism is in nature self-contained as the de-doping is electron injection induced, thus hindering "overgrowth" of the de-doped layer. In the following we shed further light on this by providing chemical proof of the de-doping mechanism. As the devices have two distinct PEDOT:PSS layers and a $\mathrm{ZnO}$ layer there are several different junctions at which the switching phenomenon could take place. Initially we believed that the switch took place at the PEDOT:PSS/ZnO junction since this was the only variation with respect to the traditional ProcessOne device structure. The solar cell stack was taken apart and built again in order to prove that the active-layer-PEDOT:PSS interface is indeed responsible for the effect. Fortunately it is facile to delaminate the solar cell exactly at the P3HT:PCBM-PEDOT:PSS interface. ${ }^{24} \mathrm{We}$ could thus prepare devices and switch them to their functional form. Delamination and removal of the PEDOT:PSS-Ag electrode followed by application of the PEDOT:PSS layer and Ag electrode, again presented a nonfunctional device, displaying the initial ohmic shunting. More importantly it could be switched anew thus unequivocally proving that the active-layer-PEDOT:PSS interface is responsible for the effect (with a series of control experiments, it was deduced that the switching was not reversed by either the exposure to vacuum or solvents). To shed further light on the de-doping mechanism in the context of the active-layer-PEDOT:PSS interface, explored here as a solar cell junction, we systematically analyzed the interfaces using chemical probe time-of-flight secondary ion mass spectrometry (TOF-SIMS). It was clear that the effect should be small if the proposed mechanism was correct since it involves only the reduction of PEDOT:PSS to the neutral semiconducting PEDOT ${ }^{0}$ and PSS-H involving water and electrons for the reduction. ${ }^{16,19}$ In terms of chemical composition, the changes at the interface are marginal and it is essentially only a redox reaction. What does change however is the polarity/ionic strength of the medium since the polythiophene cation becomes neutralized and ions are thus expected to diffuse away from the interface and towards the more ionic bulk PEDOT:PSS.

We thus analyzed the PEDOT:PSS interface through depth profiling from the surface and found a decreased sodium content at the interface for the switched device (we use sodium ions as a marker 
for the ion content). As expected the sodium content was identical in the two cases further in the bulk of the PEDOT:PSS layer. The experiment and results are shown in Fig. 3.

In order to gain appreciation for the implications and usefulness of this general approach we designed and built a machine that could usefully switch devices and modules in a full roll-to-roll process at a reasonable speed. The large current density at high fields does imply that the charge transporting layers and conductors have to dissipate heat. Whereas it is readily possible to dissipate heat when the devices are small (on the order of a few $\mathrm{mm}^{2}$ ) it is a different matter for large area devices and modules, and we found that a large drum as shown in Fig. 4d was the most rational way to cool the foil during switching.

The switched modules performed as shown in Fig. 4a and Table 1, with only a minor difference in performance between the silver and carbon based devices (depicted in Fig. 4b). This performance is comparable or better than ITO containing ProcessOne cells (ref. 6).

The switching itself is fast and takes place in a matter of milliseconds and the largest challenge for an automated setup was to ensure that all devices achieve switching simultaneously. We demonstrated that three modules, each comprising sixteen serially connected cells, could be switched in parallel (see Fig. $4 \mathrm{c}$ and d). In terms of processing speed every extra step does reduce the throughput speed. To illustrate the delay that this new process incurs, it can be rationally compared to the processing speed of the module. The complete manufacture of a single module comprising 16 serially connected stripes with the same size as a credit card takes a total of 1.2-1.7 seconds in these experiments (limited only by the length of the driers). The switching of a single module was typically achieved in $<2$ seconds (not including automated measurements of whether the switching had taken place) which is comparable to the manufacturing speed. The switching pulse duration was optimized for each roll of solar cell

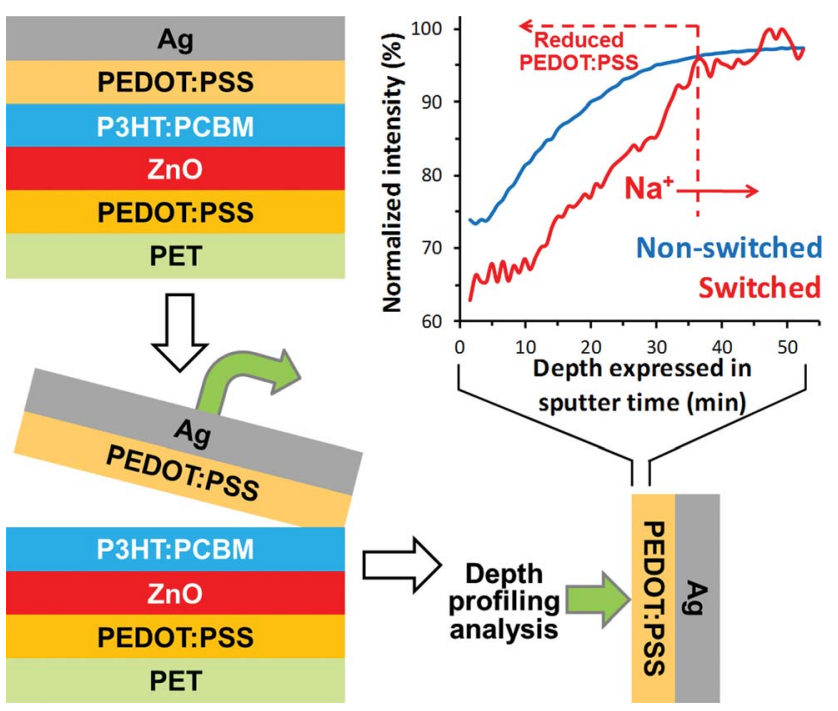

Fig. 3 Schematic OPV device illustrating where delamination occurs (confirmed from mass spectral data) that enables the exposed PEDOT:PSS surface to be analyzed. The resulting sodium ion depth profiles show a decreased content of sodium ions for the switched device in the PEDOT:PSS material facing the P3HT:PCBM-PEDOT:PSS interface.

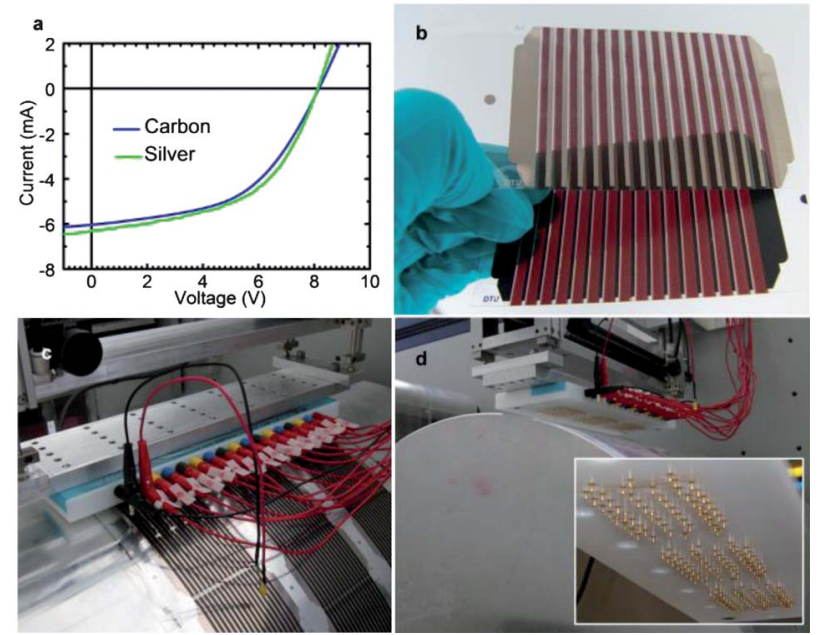

Fig. 4 (a) Current-voltage characteristics of small area modules having either a graphite or silver electrode, which are shown in the photo in (b). (c) Displays the switching setup in progress, switching 3 modules in parallel. (d) Photograph of a cooling drum and inset with close-up of gold pin array used for switching.

Table 1 Photovoltaic parameters

\begin{tabular}{lllll}
\hline Device type & PCE $(\%)^{a}$ & $J_{\mathrm{sc}}\left(\mathrm{mA} \mathrm{cm}^{-2}\right)$ & $V_{\mathrm{oc}}(\mathrm{V})$ & $\mathrm{FF}(\%)$ \\
\hline Carbon CC & 1.6 & $-6.2^{b}$ & 8.2 & 51 \\
Silver CC & 1.9 & $-6.5^{c}$ & 8.1 & 52
\end{tabular}

${ }^{a}$ Active area of the 16 striped device is $15.4 \mathrm{~cm}^{-2}$. ${ }^{b}$ Measured at $100 \mathrm{~mW}$ $\mathrm{cm}^{-2}$. ${ }^{c}$ Measured at $90 \mathrm{~mW} \mathrm{~cm}{ }^{-2}$.

modules (approx. 4900 modules for a typical run) and was typically $10 \mathrm{~ms}$. This implies that the actual speed of switching could easily reach $50 \mathrm{~ms}$ (when switching 3 modules in parallel).

In terms of applicability, we have tested this type of substrate extensively and found that it is robust in manufacture, and the switching is very consistent. It should be added that while automatic switching is a requirement for processing many modules, it is very easy to apply this principle on the laboratory scale (i.e. manual switching) and we easily foresee the use of this substrate in small research labs or even in a school classroom.

An operator can simply apply a short negative pulse by crossing two wires connected to the device. We have employed this method extensively, and it implies that the substrate described here works at all levels in terms of scale, from the student wishing to switch a small device to the professional that wish to switch multitudes of large modules in a fast and automated fashion.

\section{Conclusions}

We have described the underlying generic mechanism for this in situ formation of a charge selective interface through both chemical and physical analyses and believe that this new disruptive approach will radically change the field of polymer solar cells and finally eliminate the broad use of ITO in both research laboratories and industry. We also found that it was possible to replace silver electrodes by carbon and our modules thus represent a technology that is free from the three ingredients that hinder the wide dissemination of polymer solar 
cells, namely indium, silver and vacuum. The performance of the modules is qualitatively similar to ITO based devices with a cost reduction by a factor of $>10$ and an increase in processing speed by a factor of $>10$ under simple pilot scale conditions. In order to further the general alleviation of ITO this substrate material is made available freely by DTU to anyone with an academic interest $\uparrow$.

\section{Notes and references}

$\dagger$ Complete solar cell modules and front electrode samples comprising PET-PEDOT:PSS-ZnO are available freely for academic purposes. The authors declare no competing financial interests. Correspondence and requests for materials should be addressed to FCK.

1 L. Dou, J. You, J. Yang, C. Chen, Y. He, S. Murase, T. Moriarty, K. Emery, G. Li and Y. Yang, Nat. Photonics, 2012, 6, 180-185.

2 M. Jørgensen, K. Norrman, S. A. Gevorgyan, T. Tromholt, B. Andreasen and F. C. Krebs, Adv. Mater., 2012, 24, 580-612.

3 N. Espinosa, M. Hösel, D. Angmo and F. C. Krebs, Energy Environ. Sci., 2012, 5, 5117.

4 R. Søndergaard, M. Hösel, D. Angmo, T. T. Larsen-Olsen and F. C. Krebs, Mater. Today, 2012, 15, 36-49.

5 F. C. Krebs, S. A. Gevorgyan and J. Alstrup, J. Mater. Chem., 2009, 19, 5442.

6 F. C. Krebs, J. Fyenbo, D. M. Tanenbaum, S. A. Gevorgyan, R. Andriessen, B. van Remoortere, Y. Galagan and M. Jørgensen, Energy Environ. Sci., 2011, 4, 4116.

7 C. J. M. Emmott, A. Urbina and J. Nelson, Sol. Energy Mater. Sol. Cells, 2011, 97, 14-21.

8 F. C. Krebs, Org. Electron., 2009, 10, 761-768.

9 S. K. Hau, H.-L. Yip, J. Zou and A. K.-Y. Jen, Org. Electron., 2009, 10, 1401-1407.
10 Y. Zhou, H. Cheun, S. Choi, W. J. Potscavage, C. Fuentes-Hernandez and B. Kippelen, Appl. Phys. Lett., 2010, 97, 153304.

11 Y. Zhou, C. Fuentes-Hernandez, J. Shim, J. Meyer, A. J. Giordano, H. Li, P. Winget, T. Papadopoulos, H. Cheun, J. Kim, M. Fenoll, A. Dindar, W. Haske, E. Najafabadi, T. M. Khan, H. Sojoudi, S. Barlow, S. Graham, J.-L. Brédas, S. R. Marder, A. Kahn and B. Kippelen, Science, 2012, 336, 327-332.

12 R. Po, C. Carbonera, A. Bernardi, F. Tinti and N. Camaioni, Sol. Energy Mater. Sol. Cells, 2012, 100, 97-114.

13 F. Verbakel, S. C. J. Meskers and R. A. J. Janssen, Appl. Phys. Lett., 2006, 89, 102103.

14 U. Özgür, Y. I. Alivov, C. Liu, A. Teke, M. A. Reshchikov, S. Doğan, V. Avrutin, S.-J. Cho and H. Morkoç, J. Appl. Phys., 2005, 98, 041301.

15 S. Möller, C. Perlov, W. Jackson, C. Taussig and S. R. Forrest, Nature, 2003, 426, 166-169.

16 S. Möller, S. R. Forrest, C. Perlov, W. Jackson and C. Taussig, J. Appl. Phys., 2003, 94, 7811.

17 S. Smith and S. R. Forrest, Appl. Phys. Lett., 2004, 84, 5019.

18 B. C. de Brito, E. C. P. Smits, P. A. van Hal, T. C. T. Geuns, B. de Boer, C. J. M. Lasance, H. L. Gomes and D. M. de Leeuw, $A d v$. Mater., 2008, 20, 3750-3753.

19 J. Wang, F. Gao and N. C. Greenham, Appl. Phys. Lett., 2010, 97, 053301 .

20 J. Wang, X. Cheng, M. Caironi, F. Gao, X. Yang and N. C. Greenham, Org. Electron., 2011, 12, 1271-1274.

21 A. Manor, E. A. Katz, T. Tromholt and F. C. Krebs, Adv. Energy Mater., 2011, 1, 836-843.

22 A. Manor, E. A. Katz, T. Tromholt and F. C. Krebs, Sol. Energy Mater. Sol. Cells, 2012, 98, 491-493.

23 P.-J. Chia, L.-L. Chua, S. Sivaramakrishnan, J.-M. Zhuo, L.-H. Zhao, W.-S. Sim, Y.-C. Yeo and P. K.-H. Ho, Adv. Mater., 2007, 19, 4202-4207.

24 S. R. Dupont, M. Oliver, F. C. Krebs and R. H. Dauskardt, Sol. Energy Mater. Sol. Cells, 2012, 97, 171-175. 